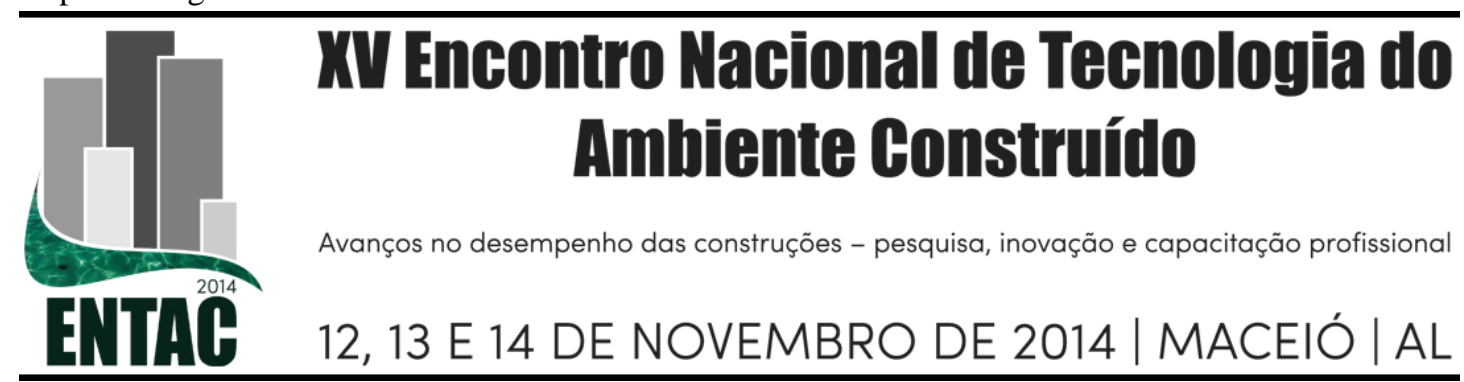

\title{
DESEMPENHO DE DIFERENTES PRATELEIRAS DE LUZ EM EDIFÍCIO COM ENTORNO CONSTRUÍDO
}

\author{
TAUFNER, Mariani (1); MAIOLI, Ricardo (2); ALVAREZ, Cristina (3)
}

(1) Universidade Federal do Espírito Santo, e-mail: marianitaufner@gmail.com, (2) Universidade Federal do Espírito Santo, e-mail: ricardomaioli@gmail.com, (3) Universidade Federal do Espírito Santo, e-mail: cristina.alvarez@ufes.br

\begin{abstract}
RESUMO
O conhecimento das prateleiras de luz sob diferentes condições permite melhor aproveitamento para a adequada distribuição da luz natural no interior dos ambientes. O objetivo dessa investigação foi simular um edifício hipotético de escritórios com a presença de prateleiras de luz curvilíneas em sua extremidade externa em comparação com prateleiras de luz planas, sob condições de obstrução externa por edifícios no entorno. Esses elementos externos bloqueiam parte da parcela visível da abóbada celeste, o que interfere na quantidade de luz admitida no ambiente interno, localizado na cidade de Vitória-ES. A análise dos níveis de iluminância foi investigada em três pavimentos com alturas diferentes, englobando situações desiguais de obstrução da abóbada. O intuito foi investigar a performance das prateleiras em situações próximas à realidade de um centro urbano, analisando a quantidade de luz admitida pelas aberturas verticais do edifício e níveis de uniformidade interna para as quatro principais orientações. A análise da admissão da luz foi baseada nos intervalos de iluminância útil (Useful daylight illuminance UDI) e a uniformidade interna. Foi usada a simulação computacional por meio do software Daysim, que calcula os valores de iluminância baseado no método Ray-tracing, considerando o clima típico local. Como conclusão observa-se que as prateleiras curvas em comparação com as planas possuem maior capacidade de redirecionamento da luz para os fundos do ambiente, principalmente nos períodos em que o ângulo de altura solar é maior. Todos os modelos simulados apresentaram um alto percentual de horas no intervalo de iluminância útil. Surpreendentemente as prateleiras obtiveram desempenho considerável nas orientações leste e oeste, em virtude da obstrução da porção inferior da abóbada pelas edificações do entorno. Esses elementos externos bloqueiam a radiação solar direta proveniente de ângulos mais baixos e as prateleiras redirecionam para o interior a luz proveniente dos maiores ângulos da trajetória solar.
\end{abstract}

Palavras-chave: Prateleira de Luz, Centro Urbano Verticalizado, Software Daysim.

\begin{abstract}
The knowledge of the device light shelf under different conditions allows better use for the proper distribution of natural light inside the rooms. The objective of this investigation was to simulate a hypothetical office building with the curvilinear lightshelves and compared to flat lightshelves, under conditions of external obstruction by nearest buildings. These external elements block the visible portion of the celestial vault, which interferes with the amount of light admitted into the room, located in Vitoria ES. The analysis of the illuminance levels was investigated in three different floors of the building, encompassing unequal situations of obstruction. The aim was to investigate the performance of the shelves in situations close to the reality of a vertical urban environment, analyzing the amount of light admitted by the vertical openings of the building and levels of internal uniformity for the four major orientations. The analysis of the admission of light was based on ranges of floor illuminance (Useful daylight illuminance - UDI) and internal uniformity. Computer simulations through Daysim software, which calculates the illuminance values based on Ray-tracing method, considering the typical local climate was used. In conclusion it is noted that the shelves curves compared to the flat have greater ability to redirect the light to the back of the room, especially in periods when the solar altitude angle is greater. All simulated models showed a high percentage of annual hours with useful illuminance.
\end{abstract}


Surprisingly, lightshelves obtained considerable performance in east and west directions, due to the obstruction of the lower portion of the dome by the buildings around. These external elements block the direct solar radiation from lower angles and shelves redirects the light from the higher angles of solar trajectory.

Keywords: Lightshelf, Vertical Urban Center, Software Daysim.

\section{INTRODUÇÃO}

Em virtude das vantagens da luz natural para o conforto visual humano em ambientes de trabalho (BOYCE, 2014), alguns sistemas de redirecionamento de luz natural, como a prateleira de luz, vem sendo estudados no campo da arquitetura como forma de aperfeiçoar o condicionamento lumínico de ambientes interiores há muitos anos, como pode ser constatado em Abdulmohsen (1994). Por possuir grande influência de controle do brilho excessivo, ocupantes que estão sob presença desse elemento sombreador demonstram menor necessidade de fechar persianas, em comparação com ocupantes em ambientes com uma janela convencional, o que contribui para economia de energia elétrica despendida com a iluminação artificial (SANATI; UTIZINGER, 2013). A necessidade de fechar persianas acontece porque o olho humano tem dificuldades de adaptação às diferenças de brilho e pode obscurecer a visão após a exposição dos olhos do usuário à luz natural direta incidente (SUK; SCHILER; KENSEK, 2013). Para minimizar esse problema, esta pesquisa trata do uso das prateleiras de luz, que fazem o redirecionamento da luz e ainda a proteção contra a radiação solar, em ambientes de escritórios.

As prateleiras de luz mais comuns possuem geometria plana e caracterizam-se por dividir a abertura vertical, responsável pelo contato externo e interno, em duas partes, inferior e superior (FREEWAN, 2010). Porém, como forma de investigar o desempenho da mesma, sob condições distintas, novas combinações vêm sendo estudadas com intuito de otimizar o uso da prateleira em diferentes situações. Freewan (2010) analisou a influência da prateleira de luz em conjunto com o teto do ambiente interno em formato curvo; Raphael (2011) simulou sistemas de prateleiras de luz automatizadas com geometria adaptável; Xue, Mak e Cheung (2014), a prateleira de luz em conjunto com o meniscus glazing panel e Maioli, Taufner e Alvarez (2014), a prateleira de luz em conjunto com diferentes tipos de vidro na parcela inferior do plano vertical.

Esse dispositivo também atua na relação de condicionamento da luz natural incidente em ambientes internos à obstrução externa. Esta é responsável por proporcionar efeitos de sombreamento da luz natural direta, devido ao bloqueio de parte da parcela da abóbada celeste e, ao mesmo tempo, propiciar reflexões de iluminâncias do entorno para o interior a ser investigado, variando de acordo com proximidade dos elementos e das cores de suas respectivas superfícies, podendo contribuir em até $26 \%$ do total. (LI; WONG, 2007; LI et al., 2006; PEREIRA; PEREIRA; CLARO, 2008).

Como ferramenta de investigação quantitativa de coeficientes de luz natural, o software Daysim demonstra precisão quanto aos níveis de iluminância durante um ano inteiro, e considera o clima típico local, baseado no método Ray-tracing (YUN; KIN, 2013). Além disso, a precisão da simulação computacional complementa-se pelo modelo de céu de Perez (1993) e pela capacidade de calcular em escala de bairros urbanos, que para aproximar-se do contexto real, leva em consideração o sombreamento e as reflexões de elementos externos modelados em geometria tridimensional detalhada (JAKUBIEC; REINHART, 2013). 


\section{OBJETIVO}

A pesquisa objetiva analisar a quantidade de luz natural admitida pelas aberturas verticais e a uniformidade alcançada nos ambientes internos presentes em um edifício comercial hipotético sob diferentes modelos de prateleiras de luz. A pesquisa comparou as prateleiras curvilíneas em sua extremidade externa com prateleiras de luz planas e com a presença de edifícios que obstruem parte da parcela visível da abobada celeste no entorno imediato, localizado na latitude $20^{\circ} 16^{\prime}$ S, cidade de Vitória - ES.

\section{METODOLOGIA}

Como metodologia de investigação, foram modeladas em $3 \mathrm{D}$ as salas comerciais e o contexto estudado por intermédio do programa computacional Google SketchUp 8.0 (GASPAR, 2010). Posteriormente, as mesmas foram exportadas para o software Daysim (REINHART, 2010) a fim de se obter os percentuais lumínicos de cada variação do modelo por meio da simulação computacional para o período de horário comercial, de $8 \mathrm{~h}$ às $18 \mathrm{~h}$ em quatro principais orientações solares em todos os dias do ano.

Após essa etapa, como meta de comparação entre os modelos propostos, foram extraídos dados de análise da admissão da luz que se baseiam nos intervalos de iluminância útil (Useful daylight illuminance - UDI), utilizados por Nabil e Mardaljevic (2006). Os níveis de iluminância são classificados como insuficientes, úteis, ou excessivos, sendo que o segundo citado abrange iluminâncias de 100 a 2000 lux. Apesar de compreender níveis abaixo dos recomendados pela NBR ISO/CIE 8995-1 (2013), de acordo com Boyce (2014), alguns ocupantes de ambientes de escritórios se sentem confortáveis mesmo submetidos em intervalos de iluminância abaixo de 500 lux. Nesse trabalho também foi avaliado o nível de uniformidade dos ambientes internos, dado pelo quociente entre a ilumiância mínima pela iluminâcia media (NBR ISO/CIE 8995-1, 2013).

$\mathrm{O}$ ambiente investigado possui dimensões de seis metros de profundidade por cinco metros de largura, baseado nas pesquisas de Bernabé (2012) e Casagrande (2013), para esse tipo de compartimento ilustrado na Figura 1. Com o intuito de investigar a captação da luz incidente, foi proposta uma malha de pontos locados nos ambientes internos englobando toda área útil, com distância de $0,5 \mathrm{~m}$ das paredes e $1 \mathrm{~m}$ entre os próprios pontos, dispostos a uma altura de $0,75 \mathrm{~m}$, simulando o plano de trabalho (NBR ISO/CIE 8995-1, 2013). 


\section{Figura 1 - Representação do ambiente investigado}

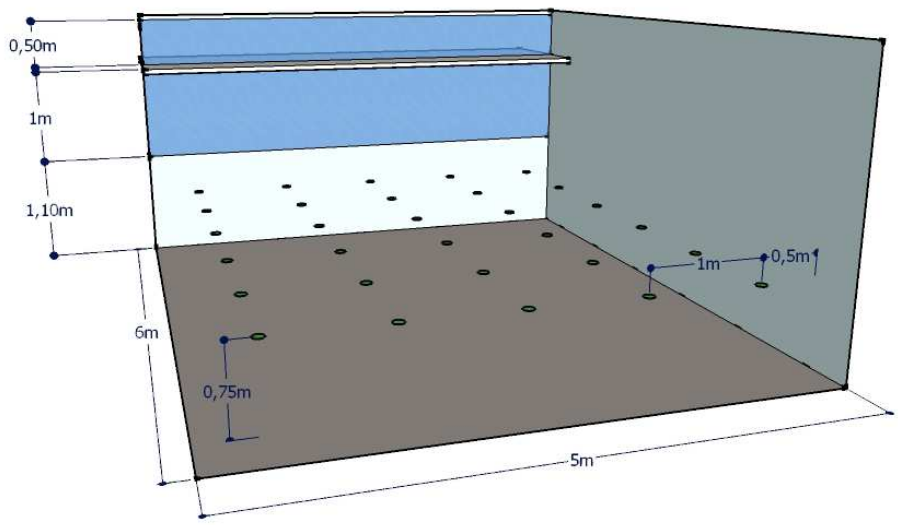

Fonte: os autores

Esta sala está inserida em um edifício de onze pavimentos, em um contexto de obstrução externa no entorno imediato. Conforme ilustrado na Figura 2, o segundo, o sétimo e o décimo pavimento dessa edificação foram os escolhidos para as simulações com o intuito de considerar diferentes situações de visibilidade do céu.

Figura 2 - Representação esquemática das obstruções
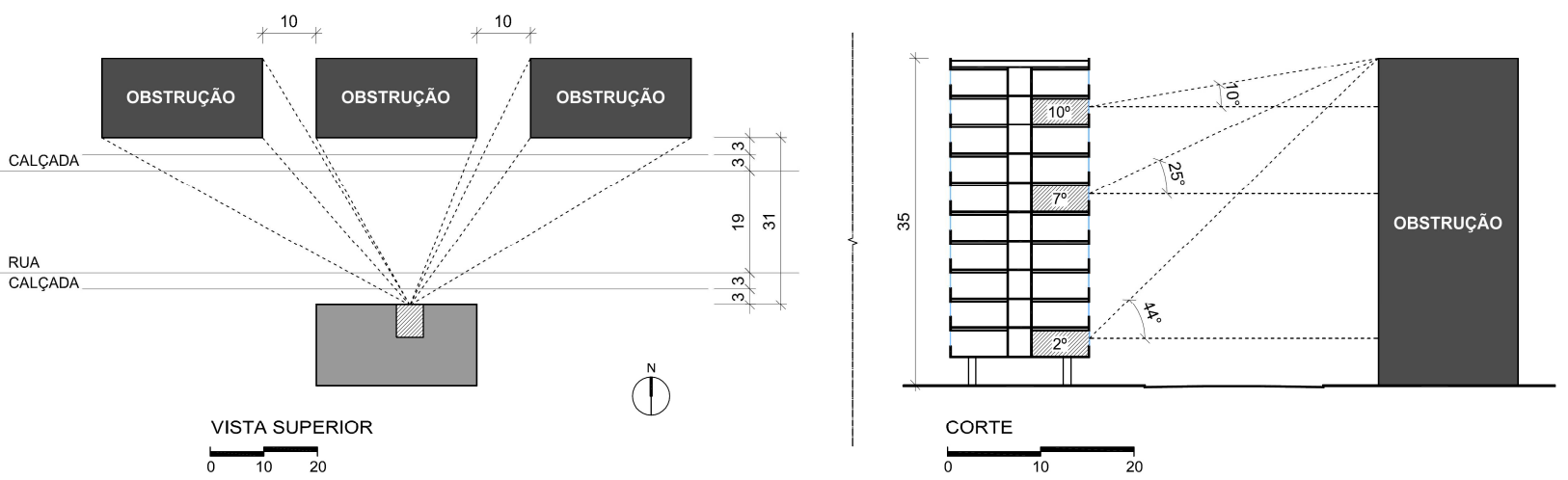

Fonte: os autores

A dimensão do dispositivo prateleira de luz está associada à trajetória solar da cidade de Vitória (LAT 20 19'10"S; LONG 40²0'16"W), com a finalidade de obstruir a radiação solar direta no interior do ambiente, presente nos horários entre $10 \mathrm{~h} 00$ e $14 \mathrm{~h} 00$ para a orientação Norte; conformando-se em um ângulo de $40^{\circ}$, como é possível observar na Figura 3. Esta mesma dimensão se repete para as outras três orientações simuladas, para fins de comparação. 


\section{Figura 3 - Representação em corte das prateleiras de luz curva ao centro,com detalhe ampliado à esquerda e representação da prateleira plana à direita.}
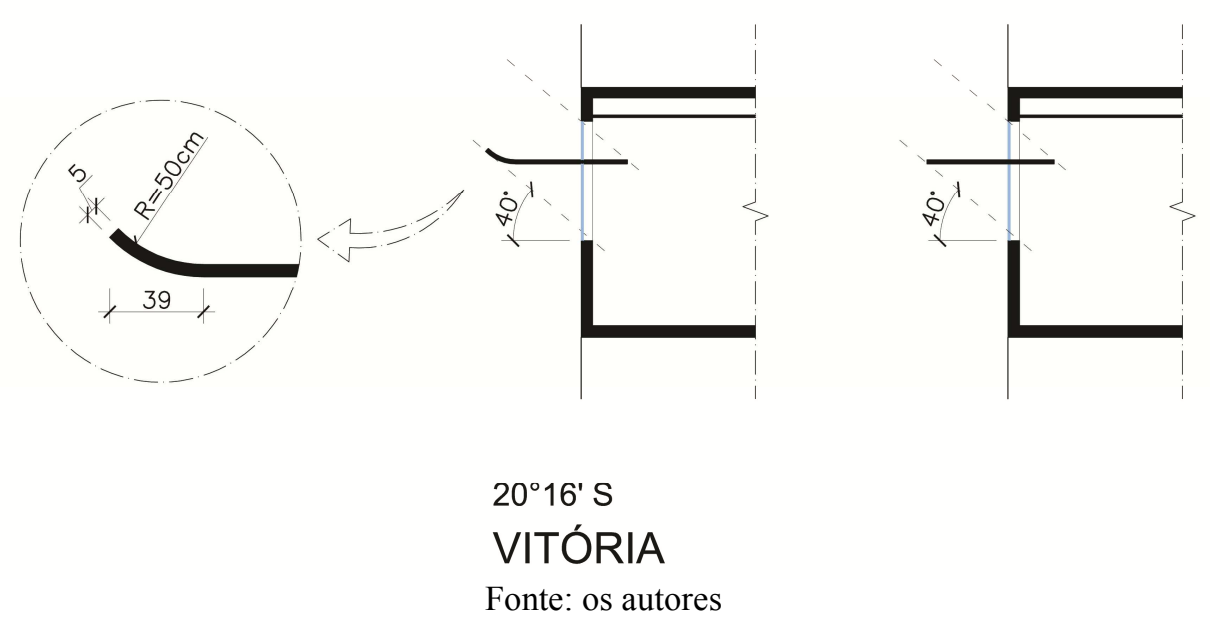

Para essa pesquisa foi considerado o uso do vidro simples nas duas porções de abertura vertical, caracterizado por possuir transmissividade de luz visível de $90 \%$ (LAMBERTS; GHISI; RAMOS, 2006). Além disso, foram considerados os valores de reflexão dos materiais, de acordo com a Tabela 1.

Tabela 1 - Porcentagem de reflexão dos materiais

\begin{tabular}{lcc}
\hline $\begin{array}{c}\text { Superfícies } \\
\text { consideradas }\end{array}$ & $\begin{array}{c}\text { Índice de reflexão } \\
\text { dos materiais }\end{array}$ & Referência \\
\hline Teto & $70 \%$ & (BERNABÉ, 2012) \\
Parede & $50 \%$ & (BERNABÉ, 2012) \\
Piso & $20 \%$ & (BERNABÉ, 2012) \\
Obstrução Externa & $40 \%$ & (CAPELUTO, 2003; LARANJA, 2009) \\
Prateleira de Luz & $80 \%$ & (MAIOLI; TAUFNER; ALVAREZ, 2014) \\
Superfície do solo & $20 \%$ & (CAPELUTO, 2003; LARANJA, 2009) \\
\hline
\end{tabular}

\section{RESULTADOS}

A análise dos dados obtidos foi desenvolvida através da elaboração de gráficos e tabelas a fim de verificar e mensurar as diferenças observadas na adoção dos dois modelos de prateleiras de luz expostas a diferentes situações.

Com o auxílio das tabelas com os valores dos intervalos da UDI, gerados pelo software Daysim, foi possível comparar os modelos e avaliar a sua capacidade de uniformização no ambiente simulado, caracterizados pela razão entre o valor mínimo e médio das iluminâncias (ABNT, 2013). Além dessa análise, também foi verificado o percentual de horas em que esses valores internos se encontram entre 100 lux e 2000 lux, o que caracterizaria um nível a ser aproveitado na iluminação interior, e nos outros dois intervalos, com iluminância excessiva e insuficiente.

\subsection{Comparação entre os modelos da fachada Norte}

Para os três pavimentos analisados, houve o aumento da porcentagem de iluminância útil de intervalo entre 100 lux e 2000 lux para a prateleira curva em comparação com a prateleira plana. Essa condição para a fachada Norte mostrou-se mais vantajosa 
principalmente no pavimento mais baixo. Quando se analisa os andares superiores, essa diferença de iluminância útil é insignificante; tornando as prateleiras curva e plana parecidas.

Para a uniformidade, os valores são bem parecidos, no entanto, a prateleira plana apresenta maior percentual no último pavimento analisado; com uma variação de $2,2 \%$ de diferença (Tabela 2).

Tabela 2 - Comparação entre os intervalos da UDI e Uniformidade - Norte

\begin{tabular}{|c|l|c|c|c|c|}
\hline \multirow{2}{*}{ Pavto. } & \multicolumn{3}{|c|}{ Intervalos da UDI } & \multirow{2}{*}{ Uniformidade } \\
\cline { 3 - 6 } & & $\begin{array}{l}\mathbf{U D I}_{<\mathbf{1 0 0}} \\
{[\mathbf{\%}]}\end{array}$ & $\begin{array}{l}\mathbf{U D I}_{\mathbf{1 0 0 - 2 0 0 0}} \\
{[\mathbf{\%}]}\end{array}$ & $\begin{array}{l}\mathbf{U D I}_{\mathbf{2 0 0 0}} \\
{[\mathbf{\%}]}\end{array}$ & \\
\hline \multirow{2}{*}{$2^{\mathbf{o}}$} & Prateleira curva & 9,2 & 86,4 & 4,4 & 0,304 \\
\cline { 2 - 6 } & Prateleira plana & 8,4 & 86,1 & 5,5 & 0,292 \\
\hline \multirow{2}{*}{$7^{\circ}$} & Prateleira curva & 5,1 & 80,7 & 14,2 & 0,239 \\
\cline { 2 - 5 } & Prateleira plana & 5,3 & 80,4 & 14,3 & 0,226 \\
\hline \multirow{2}{*}{$10^{\circ}$} & Prateleira curva & 2,7 & 76,3 & 21,0 & 0,266 \\
\cline { 2 - 5 } & Prateleira plana & 2,6 & 76,3 & 21,1 & 0,272 \\
\hline
\end{tabular}

\subsection{Comparação entre os modelos da fachada Leste}

Ao se analisar os modelos orientados para o Leste, verificou-se que o simulado com a prateleira plana só obteve maior percentual de horas com iluminância útil no pavimento mais próximo do solo, quando comparado à prateleira curva. No entanto, os valores de iluminância acima do recomendado, ou seja, acima de 2000 lux, é maior para a prateleira plana em todas as situações analisadas, conforme demonstrado na Tabela 3.

Em relação à uniformidade, a prateleira curva mostrou-se em desvantagem apenas no último andar simulado. Para os pavimentos inferiores, o valor é igual ou maior

Tabela 3 - Comparação entre os intervalos da UDI e Uniformidade - Leste

\begin{tabular}{|c|l|c|c|c|c|}
\hline \multirow{2}{*}{ Pavto. } & \multicolumn{3}{|c|}{ Modelo } & \multicolumn{3}{|c|}{ Intervalos da UDI } & \multirow{2}{*}{ Uniformidade } \\
\cline { 3 - 6 } & & $\begin{array}{l}\mathbf{U D I}_{\mathbf{1 0 0}} \\
{[\mathbf{\%}]}\end{array}$ & $\begin{array}{l}\mathbf{U D I}_{\mathbf{1 0 0 - 2 0 0 0}} \\
{[\mathbf{\%}]}\end{array}$ & $\begin{array}{l}\mathbf{U D I}_{\mathbf{2 0 0 0}} \\
{[\mathbf{\%}]}\end{array}$ & \\
\hline \multirow{2}{*}{$2^{\mathbf{o}}$} & Prateleira curva & 8,7 & 87,1 & 4,2 & 0,343 \\
\cline { 2 - 6 } & Prateleira plana & 8,1 & 87,4 & 4,5 & 0,343 \\
\hline \multirow{2}{*}{$7^{\mathbf{0}}$} & Prateleira curva & 4,8 & 81,1 & 14,1 & 0,265 \\
\cline { 2 - 6 } & Prateleira plana & 4,8 & 80,6 & 14,6 & 0,248 \\
\hline \multirow{2}{*}{$10^{\circ}$} & Prateleira curva & 3,6 & 78,8 & 17,6 & 0,255 \\
\cline { 2 - 6 } & Prateleira plana & 3,8 & 77,8 & 18,4 & 0,280 \\
\hline
\end{tabular}

\subsection{Comparação entre os modelos da fachada Sul}

Apesar da diferença de valores referentes à iluminância útil ser pequena para os três pavimentos investigados, a prateleira plana apresentou valores maiores para os andares inferiores nesta orientação. No entanto, quando se compara a uniformidade dos dois 
tipos de prateleira, a curva apresenta vantagem para as três situações. Essa diferença de valores é mais explícita para os pavimentos superiores, ou seja, a uniformidade gerada pela prateleira curva em relação à prateleira plana possui maior diferença nos pavimentos mais afastados do solo, conforme demonstrado na Tabela 4.

Tabela 4 - Comparação entre os intervalos da UDI e Uniformidade - Sul

\begin{tabular}{|c|c|c|c|c|c|}
\hline \multirow[b]{2}{*}{ Pavto. } & \multirow[b]{2}{*}{ Modelo } & \multicolumn{3}{|c|}{ Intervalos da UDI } & \multirow[b]{2}{*}{ Uniformidade } \\
\hline & & $\begin{array}{l}\text { UDI }_{<100} \\
{[\%]}\end{array}$ & $\begin{array}{l}\text { UDI }_{100-2000} \\
{[\%]}\end{array}$ & $\begin{array}{l}\mathrm{UDI}_{>2000} \\
{[\%]}\end{array}$ & \\
\hline \multirow{2}{*}{$2^{\circ}$} & Prateleira curva & 8,2 & 89,5 & 2,3 & 0,379 \\
\hline & Prateleira plana & 8,1 & 89,6 & 2,3 & 0,378 \\
\hline \multirow{2}{*}{$7^{\mathrm{o}}$} & Prateleira curva & 5,1 & 87,4 & 7,5 & 0,319 \\
\hline & Prateleira plana & 4,9 & 87,9 & 7,2 & 0,311 \\
\hline \multirow{2}{*}{$10^{\circ}$} & Prateleira curva & 2,9 & 85,8 & 11,3 & 0,318 \\
\hline & Prateleira plana & 3,0 & 85,3 & 11,7 & 0,316 \\
\hline
\end{tabular}

Fonte: os autores

\subsection{Comparação entre os modelos da fachada Oeste}

Quando se comparam os dois tipos de prateleiras para a orientação Oeste, é possível observar que a prateleira curva apresenta dados numéricos maiores tanto em uniformidade, quanto em iluminância útil. A diferença da maioria desses valores é baixa e para a situação de uniformidade do $10^{\circ}$ pavimento, é insignificante. A exceção está para a diferença de uniformidade obtida no pavimento mais baixo em comparação da prateleira curva e plana, visto que essa variação chega a 10,76\%.

Como esperado, os maiores percentuais obtidos na faixa de iluminância insuficiente foram observados nos pavimentos mais baixos; levando em consideração a obstrução da abóbada celeste (Tabela 5).

Tabela 5 - Comparação entre os intervalos da UDI e Uniformidade - Oeste

\begin{tabular}{|c|c|c|c|c|c|}
\hline \multirow[b]{2}{*}{ Pavto. } & \multirow[b]{2}{*}{ Modelo } & \multicolumn{3}{|c|}{ Intervalos da UDI } & \multirow[b]{2}{*}{ Uniformidade } \\
\hline & & $\begin{array}{l}\mathbf{U D I}_{<100} \\
{[\%]}\end{array}$ & $\begin{array}{l}\mathrm{UDI}_{100-2000} \\
{[\%]}\end{array}$ & $\begin{array}{l}\mathbf{U D I}_{>2000} \\
{[\%]}\end{array}$ & \\
\hline \multirow{2}{*}{$2^{\circ}$} & Prateleira curva & 8,7 & 88,3 & 3,0 & 0,353 \\
\hline & Prateleira plana & 8,2 & 88,2 & 3,6 & 0,315 \\
\hline \multirow{2}{*}{$7^{\circ}$} & Prateleira curva & 6,1 & 83,9 & 10,0 & 0,263 \\
\hline & Prateleira plana & 6,0 & 83,7 & 10,3 & 0,262 \\
\hline \multirow{2}{*}{$10^{\circ}$} & Prateleira curva & 3,1 & 81,8 & 15,1 & 0,282 \\
\hline & Prateleira plana & 3,1 & 81,7 & 15,2 & 0,282 \\
\hline
\end{tabular}

Fonte: os autores

\subsection{Comparação entre as orientações}

Nenhuma das duas geometrias propostas da prateleira de luz obteve unanimidade nos resultados, ou seja, nem no caso da comparação entre andares de mesma orientação, nem na comparação entre o mesmo andar em orientações diferentes. É possível observar 
esse fato ao se comparar os níveis de uniformidade para os dois tipos de prateleira analisados, sendo a situação controle a prateleira plana (Figura 4).

\section{Figura 4 - Comparação dos níveis de Uniformidade (\%)}

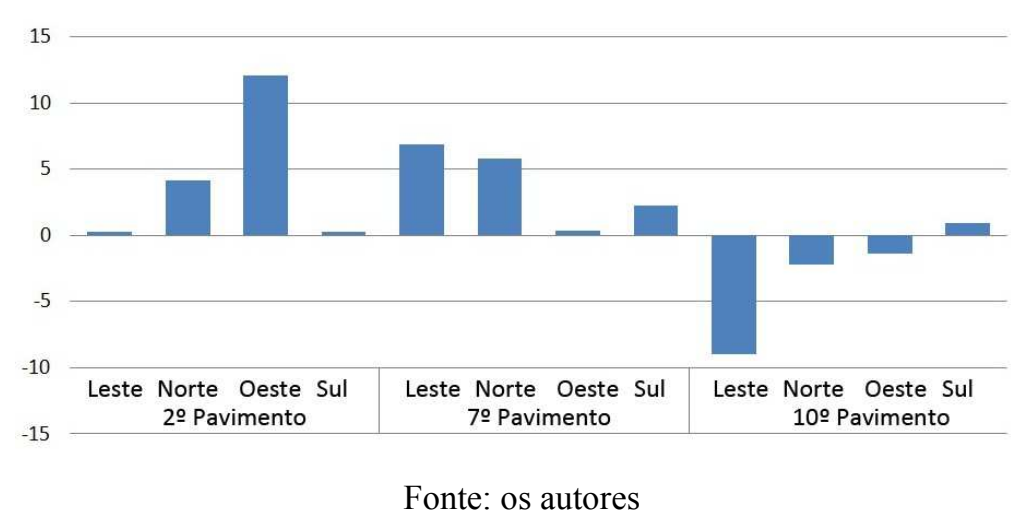

Em $75 \%$ dos resultados a prateleira curva alcançou uniformidade superior quando comparada à prateleira plana, com destaque para o segundo pavimento, orientação Oeste, com aumento de uniformidade em $12 \%$ da prateleira curva em relação à plana (Figura 4). Os resultados da prateleira plana só foram superiores para o décimo pavimento, onde a obstrução da abóbada é menor, nas orientações Leste, Norte e Oeste.

Ainda na Figura 4, é possível notar que, no segundo pavimento, as orientações Leste e Sul não apresentam variação significativa de uniformidade entre prateleira plana e curva. Essa situação se repete para a orientação Oeste, no sétimo pavimento.

O uso de prateleira de luz curva na fachada Norte mostrou-se vantajoso principalmente nos andares inferiores. Isso acontece principalmente para valores de iluminância útil. $\mathrm{O}$ contrário acontece com a fachada Leste, onde a prateleira curva é a responsável por maior quantidade de iluminância útil nos pavimentos superiores. Em contrapartida, os valores de uniformidade mostram-se em desvantagem sob essa mesma condição.

Para essas mesmas orientações, Norte e Leste, os percentuais observados dos valores com iluminâncias acima de 2000 lux são maiores em relação às outras duas orientações, e atingem valores parecidos nos andares mais baixos.

As orientações Sul e Oeste apresentam, respectivamente, os maiores valores de iluminância útil quando comparados com as demais orientações.

Em contrapartida, a fachada Norte apresentou os menores percentuais de horas no intervalo de iluminância útil. Nesta orientação foram observados os maiores valores com iluminância excessiva e os menores com iluminância insuficiente. Essa observação deve-se ao fato da fachada Norte apresentar o maior número de horas com incidência de radiação solar direta dentre todas as orientações. Somado a isso, o vidro utilizado na porção inferior das prateleiras possui um alto índice de transmitância visível, o que contribui para o número elevado de horas observadas acima de 2000 lux.

$\mathrm{Na}$ fachada Norte foram verificados quase todos os piores resultados de uniformidade, quando comparados os mesmos dispositivos nas quatro orientações. O menor índice de uniformidade foi observado na orientação Norte, no $7^{\circ}$ pavimento, enquanto o maior índice foi registrado na fachada Sul, no $2^{\circ}$ pavimento (Figura 5). 
Figura 5 - Representação das médias por horas de sol dos valores de iluminância para melhor e pior situação de uniformidade, 0.379 e 0.226 , respectivamente.
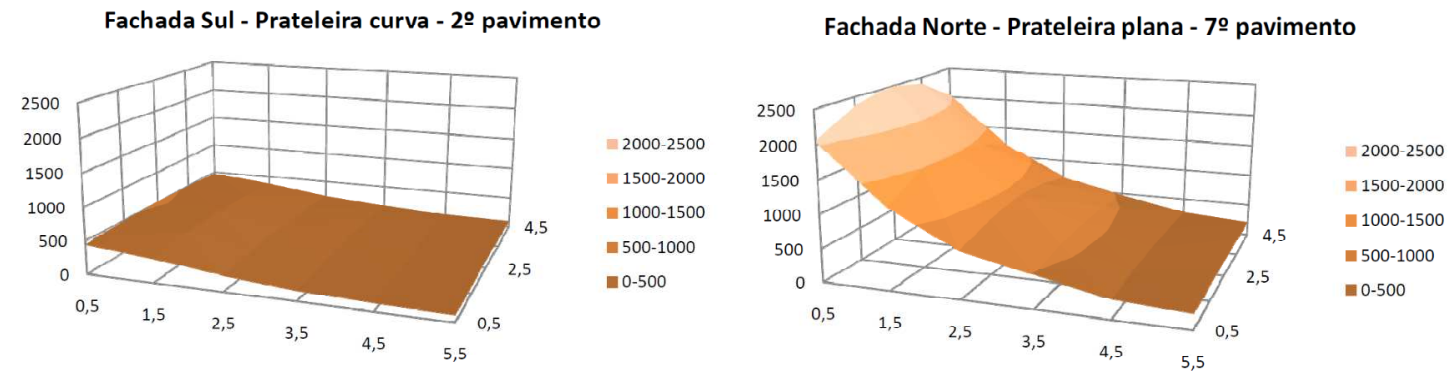

Fonte: os autores

\section{CONCLUSÕES}

Pode-se notar que valores de iluminância útil para todas as orientações, independente do tipo de prateleira estudada, é inversamente proporcional à altura do pavimento analisado, ou seja: quanto mais baixo o pavimento, maior o valor de iluminância útil. Credita-se isso ao fato dos primeiros pavimentos apresentarem um maior percentual de área obstruída da abóbada celeste, diminuindo os valores observados de iluminâncias excessivas. Essa situação não se repete para valores de uniformidade, ou seja, para as orientações Norte e Oeste, por exemplo, os valores de uniformidade para prateleiras planas e curvas são menores para o pavimento intermediário.

Com os resultados obtidos, é notória a influência das variáveis simuladas em relação à condição submetida de disponibilidade de luz natural no espaço interno. No caso dos modelos simulados, a obstrução dos edifícios do entorno auxiliam na diminuição da radiação solar proveniente de ângulos menores, contribuindo para um bom desempenho das prateleiras em orientações incomuns para o uso desse dispositivo, como a Leste e a Oeste.

Nos casos de prateleiras de luz curva, a vantagem em relação à prateleira plana, quanto à iluminância útil e uniformidade, se destaca de acordo com a orientação selecionada e, também, de acordo com o pavimento analisado.

\section{REFERÊNCIAS}

ABDULMOHSEN, A.; BOYER, L. L.; DEGELMAN, L. O. Evaluation of lightshelf daylighting systems for office buildings in hot climates. In: proceedings of the ninth symposium on improving building systems in hot and humid climates, Arlington, TX; 1994.

ASSOCIAÇÃO BRASILEIRA DE NORMAS TÉCNICAS (ABNT). NBR ISO/CIE 8995-1: Iluminação de ambientes de trabalho. Parte1: Interior, 2013.

BERNABÉ, A. C. A. A influência da envoltória no consumo de energia em edifícios comerciais artificialmente climatizados na cidade de Vitória-ES. 2012. 130p. Dissertação de Mestrado - Universidade Federal do Espírito Santo, Vitória, 2012.

BOYCE, P. R. Human Factors in lighting. 3.ed. New York: Taylor \& Francis Group, 2014.

CASAGRANDE, B. G. Cenários climáticos futuros: diagnóstico prospectivo do desempenho termo energético de edifícios comerciais no Brasil para o século XXI. 2013. 135p. Dissertação de Mestrado - Universidade Federal do Espírito Santo, Vitória, 2013. 
FREEWAN, A. A. Maximizing the lightshelf performance by interaction between lightshelf geometries and a curved ceiling. Energy Conversion and Management, n.51, p.1600-1604. 2010.

GASPAR, J. A. M. Google SketchUp Pro 8 passo a passo (em português). São Paulo: [s.n.], 2010.

JAKUBIEC, J. A.; REINHART, C. F. A method for predicting city-wide electricity gains from photovoltaic panels based on LiDAR and GIS data combined with hourly Daysim simulations. Solar Energy, v.93, p. 127-143. 2013.

LAMBERTS, R.; GHISI, E.; RAMOS, G. Impactos da adequação climática sobre a eficiência energética e o conforto térmico de edifícios de escritórios no Brasil. Florianópolis: LABEEE, 49p. 2006.

LI, D. H. W.; WONG, S. L. Daylighting and energy implications due to shading effects from nearby buildings. Applied Energy, v.84(12), p.1199-1209. 2007.

LI, D. H.W.; WONG, S. L.; TSANG, C.L.; CHEUNG, G.H.W. A study of the daylighting performace energy use heavily obstructed residencial building via computer simulation. Energy and Buildings, v. 38(11), p. 1343-1348. 2006.

MAIOLI, R. N.; TAUFNER, M. D.; ALVAREZ, C. E. Impacto da escolha do vidro em aberturas associado ao dispositivo prateleira de luz para o conforto visual em ambientes de escritório. In: Congresso Luso-Brasileiro de Materiais de Construção Sustentáveis, 2014, Guimarães. Materiais de Construção Sustentáveis. Guimarães: Universidade do Minho, v. 3. p. 197-206. 2014.

NABIL, A.; MARDALJEVIC, J. Useful daylight illuminances: A replacement for daylight factors. Energy and Buildings, n.38, p.905-913, 2006.

PEREIRA, R. C.; PEREIRA, F. O. R.; CLARO, A. Caracterização da contribuição do entorno na avaliação da iluminação natural em edificações. Ambiente Construído, Porto Alegre, v. 8, n. 4, p. 103-115. 2008.

PEREZ, R.; SEALS, R.; MICHALSKY, J. All-weather model for sky luminance distributionPreliminary configuration and validation. Solar Energy, v.50, n.03, p.235-245. 1993.

RAPHAEL, B. Active control of daylighting features in buildings. Computer-Aided Civil and Infrastructure Engineering, n.26, p.393-405, 2011.

REINHART, C. F. Tutorial on the Use of Daysim Simulation for Sustainable Design. Havard Design School, Cambridge. 2010.

SANATI, L.; UTIZINGER, M. The effect of window shading design on occupant use of blinds and electric lighting. Building and Environment, v.64, p.67 (10). 2013.

SUK, J. Y.; SCHILER, M.; KENSEK, K. Development of new daylight glare analysis methodology using absolute glare factor and relative glare factor. Energy and Buildings, v.64, p.113-122. 2013.

YUN, G.; KIM, K. S. An empirical validation of lighting energy consumption using the integrated simulation method. Energy and Building, v.57, p. 144-154. 2013.

XUE, P.; MAK, C. M.; CHEUNG, H. D. New static lightshelf system design of clerestory windows for Hong Kong. Building and Environment, v.72, p. 368-376. 2014. 